\title{
Avaliação da prevenção da transmissão vertical do HIV em Belo Horizonte, MG, Brasil
}

\author{
Prevention evaluation of HIV vertical transmission in Belo Horizonte, MG, Brazil \\ Evaluación de la prevención de la transmisión vertical del VIH en Belo Horizone, MG, Brasil
}

\section{Francisco Carlos Felix Lana', Alessandra Silva Lima'}

'Universidade Federal de Minas Gerais. Departamento de Enfermagem Materno-Infantil e Saúde Pública. Belo Horizonte, MG

Submissão: 05/04/2009

Aprovação: 09/07/2010

\section{RESUMO}

A pesquisa teve como objetivo analisar o pré-natal ofertado na rede básica de saúde do Distrito Leste do município de Belo Horizonte e as ações direcionadas à prevenção da transmissão vertical do HIV. Foi discutida a permanência de taxas significativas de transmissão do HIV na vigência de tecnologias adeQuadas para a sua prevenção. Por meio de uma análise descritiva e univariada, Que abordou os bancos de dados Sisprenatal e Sinan, identificou-se entraves Que se relacionam diretamente a problemas observados na captação precoce de gestantes e na instituição da terapêutica em tempo adequado. $\mathrm{O}$ envolvimento dos gestores e a capacitação dos profissionais envolvidos é essencial para o correto direcionamento de ações Que possibilitem a prevenção efetiva da transmissão vertical do HIV.

Descritores: Infecções por HIV; Transmissão vertical de doença infecciosa; Avaliação de serviços de saúde; Cuidado pré-natal; Enfermagem.

\section{ABSTRACT}

The study had as objective to analyze the prenatal care services offered by the East District's primary healthcare network of the city of Belo Horizonte as well as actions for the prevention of vertical transmission of HIV. It discussed the still significant rates of transmission of HIV in the presence of appropriate technologies for its prevention. Barriers directly related to problems in the early uptake of pregnant women and the institution of appropriate therapy in time were identified through univariate descriptive analysis considering Sisprenatal and Sinan databases. The involvement of managers and the training of professionals are essential for the correct direction of actions that enable the effective prevention of vertical transmission of HIV.

Key words: HIV Infections; Infectious disease transmission, vertical; Health services evaluation; Prenatal care; Nursing.

\section{RESUMEN}

Este trabajo de investigación tuve como objetivo analizar la atención prenatal de la red básica del Distrito Este del municipio de Belo Horizonte y las acciones Que buscan prevenir la transmisión del VIH de madre a hijo. Se discutió el porqué de las altas tasas de transmisión vertical de dicho virus cuando hay tecnologías adecuadas para su prevención. Se realizó un análisis descriptivo y univariado de información de los bancos de datos Sisprenatal y Sinan. Se identificaron obstáculos para los problemas observados en la captación temprana de embarazadas y en la institución de la terapéutica en tiempo adecuado. El compromiso de los gestores y la capacitación de los profesionales son esenciales para orientar acciones de prevención efectiva de la transmisión vertical del VIH.

Descriptores: Infecciones por VIH; Transmisión vertical de enfermedad infecciosa; Evaluación de servicios desalud; Atención prenatal; Enfermería. 


\section{INTRODUÇÃO}

A transmissão vertical se tornou, no decorrer dos anos, a principal via de infecção do HIV em crianças. A primeira ocorrência de transmissão vertical registrada no Brasil se deu em 1985, no estado de São Paulo, onde foram diagnosticados dois pacientes, Que representavam $0,4 \%$ do total de casos do período. No ano de 2006, foi responsável por $85,2 \%$ dos casos em menores de 13 anos de idade, e em 2007, por 91,4\% do total de $\operatorname{casos}^{(1)}$.

Em 1994, os resultados de um estudo realizado nos EUA e França, com gestantes HIV positivas, comprovaram Que o uso da zidovudina (AZT) a partir da $14^{\mathrm{a}}$ semana de gestação, no momento do parto e na criança, até a $6^{\text {a }}$ semana de vida, reduzia em $67,5 \%$ o risco de transmissão perinatal do $\mathrm{HIV}^{(2)}$. Este estudo recebeu o nome de Protocolo 076 do Aids Clinical Trial Group (ACTG-076) e significou um avanço no manejo da transmissão vertical. O estudo mencionado afirma Que, sem nenhuma intervenção, a taxa de transmissão vertical do HIV situa-se em torno de $20 \%$.

Estudos comprovaram a redução da carga viral plasmática para níveis indetectáveis com o uso da terapia antiretroviral combinada ${ }^{(3,4)}$. Atualmente, o Ministério da Saúde preconiza o esquema duplo para o tratamento e, caso a gestante possua carga viral superior a 10.000 cópias/ml, é instituída a terapia tripla, a fim de reduzir a possibilidade de resistência aos antiretrovirais (ARV) ${ }^{(5)}$.

No entanto, foi constatado Que o uso de antiretrovirais durante a gestação não contempla todas as gestantes soropositivas ${ }^{(6)}$. A ausência da utilização dos ARV por essas gestantes se reflete diretamente em taxas de transmissão vertical do HIV ainda significativas ${ }^{(1)}$.

Essa cobertura ainda insatisfatória, mesmo dispondo de tecnologia adequada, ou seja, o acesso aos ARV, leva a uma reflexão em relação à Qualidade da assistência prestada a essas gestantes durante o pré-natal. O processo de vigilância a saúde possui falhas, o Que pode ser demonstrado pela ausência de captação precoce e busca ativa de gestantes Que faltam à consulta de pré-natal ${ }^{(7)}$. A captação tardia caracterizada pelo início do pré-natal após a $16^{\mathrm{a}}$ semana de gestação reduz a eficácia de ações preventivas, uma vez Que a terapia antiretroviral disponibilizada durante o período gestacional não será iniciada a partir da $14^{\mathrm{a}}$ semana de gestação, o Que pode resultar na infecção do concepto.

O objetivo desse estudo é analisar o pré-natal ofertado nas unidades básicas de saúde e as ações direcionadas à prevenção da transmissão vertical do HIV.

\section{MÉTODO}

Trata-se de um estudo descritivo-exploratório, Que se insere no campo das pesquisas avaliativas em saúde. O processo de avaliação envolve um julgamento de valor a respeito de uma intervenção, ou sobre QualQuer um de seus componentes, visando auxiliar na tomada de decisões ${ }^{(8)}$.

O objeto, estudo dessa pesQuisa avaliativa, é a prevenção da transmissão vertical do HIV no Distrito Leste de Belo Horizonte. O enfoque recai sobre a análise da intervenção do programa de prénatal ofertado pela rede básica de saúde, com ênfase nas ações preventivas relacionadas à transmissão vertical do HIV.

O estudo foi realizado a partir da análise de dados secundários pertencentes ao Distrito Sanitário Leste (Disal). O Distrito Leste é composto por Quinze centros de saúde e dois serviços de atenção secundária com diversas especialidades. Possui um Laboratório Distrital, um Centro de Referência para Saúde Mental (Cersam) e um Centro de Testagem e Aconselhamento (CTA) Que presta serviços e orienta sobre a Aids e as doenças sexualmente transmissíveis. Aproximadamente $70 \%$ da população é usuária do Sistema Único de Saúde ${ }^{(7)}$. O Disal foi escolhido para a pesquisa por ter sido pioneiro na implantação do Projeto Vida, Que se constituiu em uma estratégia de reorganização da assistência materno-infantil, implantada em 1993, em Belo Horizonte. Além disso, a organização das informações sobre o binômio mãe-filho é realizada em tempo superior ao das outras regionais, com maior "status" de processamento organizacional das informações, o que facilitou a coleta e a utilização dos dados.

Foram utilizados como fontes de informação os relatórios gerenciais do banco de dados do Ministério da Saúde Sisprenatal e as Fichas de Notificação de Gestantes HIV Positivas e Crianças Expostas, pertencentes ao Sinan.

O Sisprenatal é um banco de dados criado para o gerenciamento do Programa de Humanização no Pré-Natal e Nascimento (PHPN), com o objetivo de fornecer relatórios e indicadores da Qualidade da assistência do pré-natal(9).

Os indicadores do Sisprenatal selecionados para o estudo foram os seguintes: percentual de gestantes Que se inscreveram no programa e realizaram a primeira consulta (cobertura); percentual de gestantes que se inscreveram no Programa e realizaram a primeira consulta em até 120 dias (cobertura de captação precoce); percentual de gestantes inscritas Que realizaram seis consultas de pré-natal e que tenham, pelo menos, 240 dias de idade gestacional; percentual de gestantes inscritas Que realizaram seis consultas de pré-natal e a consulta de puerpério; percentual de gestantes inscritas Que realizaram seis consultas de pré-natal e todos os exames básicos e Que possuam, pelo menos, 240 dias de idade gestacional; percentual de gestantes inscritas Que realizaram o teste anti-HIV e Que possuam 280 dias ou menos de idade gestacional.

O Sinan consiste em um sistema de informação, alimentado principalmente, pela notificação e investigação de casos de doenças e agravos Que constam na lista nacional de doenças de notificação compulsória (Portaria GM/MS no 33 de 14 de julho de 2005) (10). $^{(10}$. $\mathrm{O}$ agravo abordado no estudo foi infecção pelo HIV em gestantes e crianças expostas ao risco de transmissão vertical. Segundo a Portaria Ministerial $n^{0} 2.325$, de oito de dezembro de 2003, é obrigatória a notificação de todas as gestantes HIV positivas e de todas as crianças, filhas de mães soropositivas ou Que tenham sido amamentadas por mulheres HIV positivas( ${ }^{(1)}$. A notificação é realizada em serviços de pré-natal, maternidades, ambulatórios materno-infantis e serviços de referência para DST/Aids, por meio da Ficha de Investigação Gestante HIV positiva e crianças expostas.

As variáveis a serem utilizados no estudo extraídas dessa ficha de investigação são os seguintes: idade da gestante no momento da evidência laboratorial do HIV; grau de escolaridade da gestante; evidência laboratorial do HIV (antes do pré-natal, durante o prénatal, durante o parto, após o parto); idade gestacional (em semanas) na primeira consulta de pré-natal; em uso de antiretrovirais para tratamento (sim, não, ignorado); se realizada profilaxia com antiretrovirais, idade gestacional em Que foi iniciada; se não foi 
realizada profilaxia $A R V$, motivos (atraso na entrega do resultado à gestante, ignorado, recusa da gestante, ARV não oferecido, antiretroviral não disponível, outros motivos).

É importante relatar Que, para uma melhor compreensão acerca dos dados do Distrito Sanitário Leste, também foram coletados os indicadores do banco Sisprenatal do município de Belo Horizonte, a fim de estabelecer comparações entre os resultados alcançados pelo município e pelo Distrito.

A coleta dos dados referentes ao Distrito Leste incluiu os anos de 2004 e 2005. Além dos bancos de dados, foram consultados documentos e relatórios provenientes da Área Técnica da Saúde da Mulher do Ministério da Saúde e da Secretaria Municipal de Saúde de Belo Horizonte.

Para o tratamento dos dados provenientes dos sistemas de informação, foi utilizado o Epi-Info versão 3.2. Realizou-se uma análise descritiva, univariada, Que abordou a distribuição de freQüências simples para as variáveis citadas, com a apresentação dos dados na forma de tabelas e gráficos em barras.

O presente trabalho teve início somente após sua avaliação e aprovação pelos Comitês de Ética em pesquisa da Universidade Federal de Minas Gerais (Parecer no 512/05) e da Secretaria Municipal de Saúde de Belo Horizonte (Protocolo $n^{\circ}$ 03/2006). Todas as informações coletadas no período determinado não continham a identificação de indivíduos ou instituições, mantendo, portanto, a confidencialidade em relação às fontes de informação.

\section{RESULTADOS}

A análise dos dados secundários nos possibilitou a identificação de inúmeros entraves na realização de um pré-natal de Qualidade. Em relação aos indicadores referentes ao Sisprenatal, os percentuais encontram-se nas Tabelas 1 e 2.
Em relação à cobertura de gestantes, o indicador é obtido dividindo-se o número de gestantes Que se inscreveram no programa e realizaram a primeira consulta pelo número de nascidos vivos do período, multiplicado por 100 . O indicador do município sofreu decréscimo do ano 2004 (65, I\%) para 2005 (59,3\%), enQuanto no Distrito Leste permaneceu praticamente o mesmo.

O indicador de captação precoce é obtido dividindo-se o número de gestantes que se inscreveram no programa e realizaram a primeira consulta pré-natal em até 120 dias pelo número de nascidos vivos do período, multiplicado por 100. Em 2004, no Distrito Leste, obteve-se um percentual de gestantes com captação até 120 dias de gestação da ordem de 40,6\%, e em 2005, 39,6\%. Em relação a Belo Horizonte, foram captadas precocemente, em 2004, 15.363 gestantes e, em 2005, 13.790 gestantes, totalizando percentuais de $46,8 \%$ e $43,1 \%$, respectivamente, o Que demonstra Queda na captação.Em relação ao percentual de gestantes Que realizaram 6 consultas de pré-natal, o Distrito Leste se destacou com um incremento do número de gestantes com 6 consultas durante o pré-natal: em 2004, $21,5 \%$ das gestantes, e em 2005, um percentual de $26,9 \%$.

Analisando-se o indicador Que conjuga as 6 consultas de prénatal associada à realização da consulta de puerpério, foi observado no distrito, um pequeno aumento desse percentual de 2004 para 2005 , gerando percentuais de $6,2 \%$ e 10,2\%, respectivamente.

Em relação ao indicador percentual de gestantes inscritas Que realizaram as 6 consultas de pré-natal e todos os exames básicos, o distrito também apresentou um aumento desse indicador. Em 2004, foram 195 gestantes, 17,4\%; e em 2005, foram 231 gestantes, correspondendo a $21,9 \%$.

Em relação ao indicador percentual de gestantes inscritas Que realizaram o teste anti-HIV, observou-se pouca variação nos resultados dos anos analisados. Em relação ao Distrito Leste, houve

Tabela 1. Indicadores do Sisprenatal, Distrito Leste. Belo Horizonte, 2004.

\begin{tabular}{|c|c|c|c|c|}
\hline \multirow{2}{*}{ Indicadores } & \multicolumn{2}{|c|}{ Distrito Leste } & \multicolumn{2}{|c|}{ Belo Horizonte } \\
\hline & $\mathrm{n}$ & $\%$ & $\mathrm{n}$ & $\%$ \\
\hline Cobertura de gestantes & 1956 & 57,1 & 21354 & 65,1 \\
\hline Captação precoce & 1390 & 40,6 & 15363 & 46,8 \\
\hline Seis consultas mínimas & 235 & 21,5 & 3659 & 28,0 \\
\hline Seis consultas de pré-natal e a consulta de puerpério & 20 & 6,2 & 441 & 11,2 \\
\hline Seis consultas de pré-natal e todos os exames básicos & 195 & 44,0 & 151 & 16,4 \\
\hline Realização do teste anti HIV & 406 & 44,0 & 4750 & 45,6 \\
\hline
\end{tabular}

Fonte: Relatório de Indicadores Sisprenatal - Gersa (Leste e SMSA-BH).

Tabela 2. Indicadores do Sisprenatal, Distrito Leste. Belo Horizonte, 2005.

\begin{tabular}{|c|c|c|c|c|}
\hline \multirow{2}{*}{ Indicadores } & \multicolumn{2}{|c|}{ Distrito Leste } & \multicolumn{2}{|c|}{ Belo Horizonte } \\
\hline & $\mathrm{n}$ & $\%$ & $\mathrm{n}$ & $\%$ \\
\hline Cobertura de gestantes & 1844 & 57,2 & 18963 & 59,3 \\
\hline Captação precoce & 1276 & 39,6 & 13790 & 43,1 \\
\hline Seis consultas mínimas & 301 & 26,9 & 3282 & 27,1 \\
\hline Seis consultas de pré-natal e a consulta de puerpério & 32 & 10,2 & 429 & 11,9 \\
\hline Seis consultas de pré-natal e todos os exames básicos & 231 & 21,9 & 2074 & 17,2 \\
\hline Realização do teste anti HIV & 313 & 44,5 & 4120 & 47,2 \\
\hline
\end{tabular}


pouca variação, constatada por $44,0 \%$ das gestantes submetidas à testagem do HIV e, no ano de 2005 , foram $44,5 \%$ das gestantes. No município de Belo Horizonte, em 2004, a porcentagem foi de 45,6\% das gestantes com sorologia anti-HIV e, em 2005, 47,4\%.

Em relação às gestantes soropositivas para o HIV, analisando-se Ficha de Investigação Gestante HIV positiva e crianças expostas, foram identificadas 14 gestantes soropositivas em 2004 e 15 soropositivas em 2005, chegando um total de 29 gestantes HIV positivas notificadas no Distrito Leste neste período. Em relação à idade dessas mulheres soropositivas, os dados demonstram um maior acometimento das faixas etárias de 25 a 29 anos (27,6\%), 30 a 34 anos $(20,7 \%)$ e 35 a 39 anos $(20,7 \%)$, demonstradas no Figura 1 .

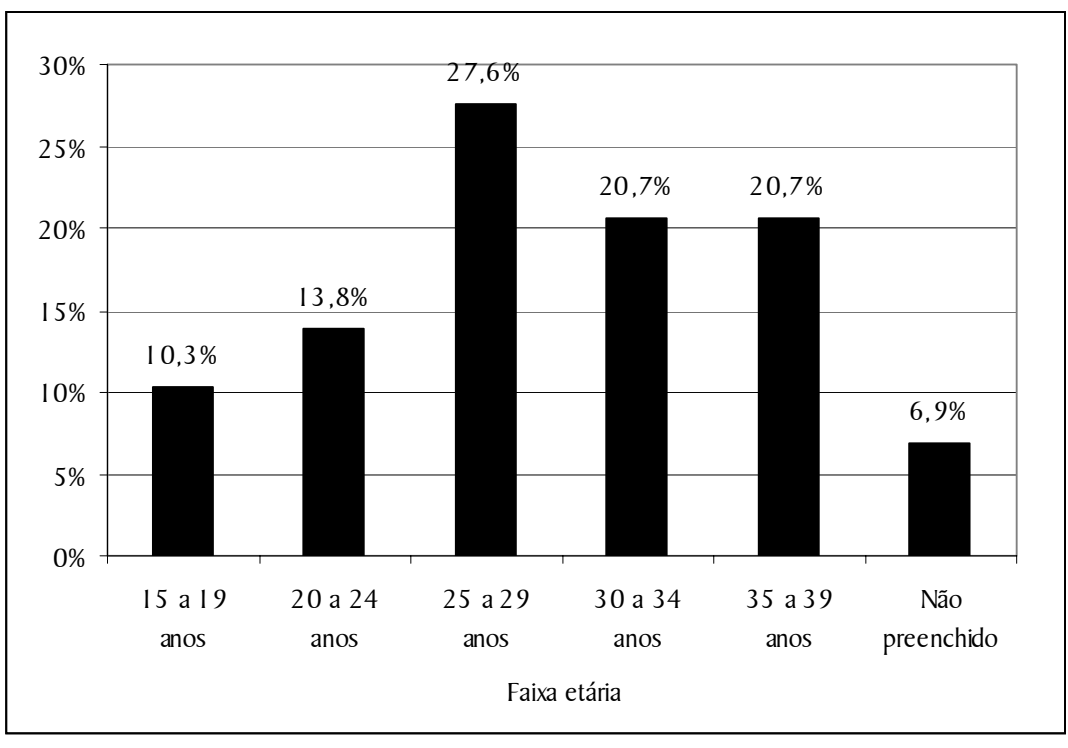

Figura I. Faixa etária das gestantes HIV positivas no Distrito Leste. Belo Horizonte, 2004-2005.

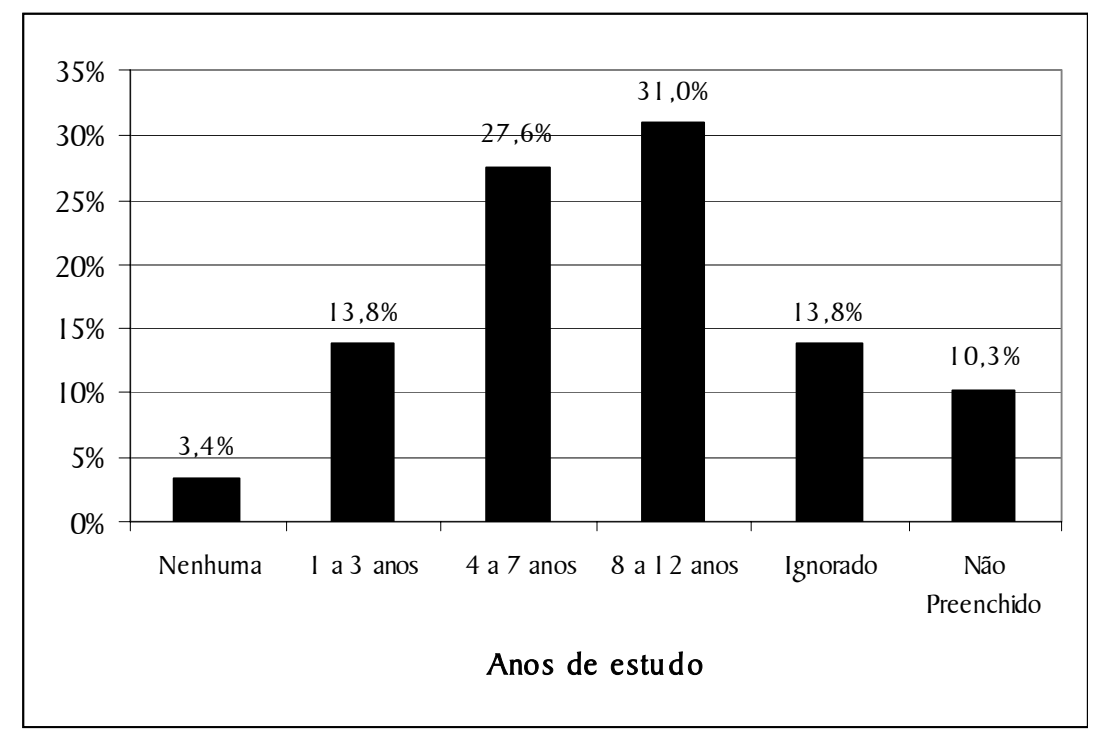

Figura 2. Anos de estudo das gestantes HIV positivas do Distrito Leste. Belo Horizonte, 2004-2005.
Com relação à escolaridade, identificamos um grande percentual de mulheres com baixa escolaridade, conforme demonstrado no Figura 2.

Excluindo-se os casos ignorados (13,8\%) e os campos de escolaridade não preenchidos da ficha de notificação $(10,3 \%)$, o Que impossibilitou delimitar o grau de escolaridade de sete gestantes, observou-se, Que mulheres com baixa escolaridade, ou seja, com menos de oito anos de estudo, somam $44,8 \%$ dos casos notificados no Distrito Leste, enquanto mulheres com 8 a 12 anos de estudo correspondem a 31\%. É importante mencionar Que o campo 12 anos ou mais de estudo não foi incluído no gráfico, devido à ausência de gestantes com esse grau de escolaridade nos anos analisados.

Em relação ao momento em Que a mulher conheceu seu "status" sorológico, identificou-se uma alta porcentagem de mulheres sabidamente soropositivas para o vírus, antes da gestação, correspondendo a uma faixa de $48,3 \%$. Um total de $20,6 \%$ das gestantes não obteve acompanhamento pré-natal adequado, tendo sido revelada a sorologia positiva para o HIV durante o parto ou após o parto (Figura 3).

Um total de 18 mulheres HIV positivas fizeram uso dos ARV durante o pré-natal, sendo Que 14 mulheres já conheciam seu "status" sorológico antes de engravidarem e Quatro mulheres obtiveram o diagnóstico durante a gestação (Tabela 3).

Desse total de mulheres com sorologia comprovada para o HIV antes do pré-natal, podemos notar Que oito mulheres usavam os ARV antes da gestação e seis mulheres iniciaram os ARV durante o pré-natal.

A média de IG de captação para as mulheres sabidamente soropositivas antes da gestação e Que já usavam ARV foi de 13,3 semanas para o início do pré-natal. Enquanto para as mulheres também soropositivas antes da gestação, mas Que não usavam ARV foi de 21 semanas, e para as mulheres diagnosticadas durante o pré-natal a média foi 22,5 semanas.

Em relação à idade gestacional de início dos ARV, também foi comprovada uma diferença nas médias: para as mulheres soropositivas antes do pré-natal, a média foi de 23,3 semanas para o início dos ARV, e uma média de 25,5 semanas para o início dos ARV para as mulheres Que obtiveram o resultado da soropositividade durante o pré-natal.

Um total de apenas 18 mulheres $(62,1 \%)$ realizou profilaxia com ARV durante o período gestacional, ou seja, 1 I mulheres não obtiveram acesso à profilaxia da prevenção da transmissão vertical do HIV. Em relação às razões Que levaram a não execução da terapia antiretroviral, destacouse na ficha de notificação a não realização do pré-natal. Um total de cinco mulheres soropositivas $(45,5 \%)$ não realizou pré-natal, o 
Que levou a não utilização dos ARV durante a gestação.

Uma gestante $(9,1 \%)$ Que realizou o pré-natal na rede básica, entrou em trabalho de parto sem o uso dos ARV durante a gestação, devido ao atraso na entrega do resultado do teste anti-HIV.

O dado ignorado, segundo a ficha de notificação, se relaciona a uma gestante soropositiva $(9,1 \%)$ Que realizou o pré-natal em consultório particular, não constando o uso dos ARV em sua ficha. Nesse caso, é desconhecida a conduta adotada, pois, ao Que parece, perdeuse o contato com essa gestante, e, assim, não se sabe se ela fez uso dos ARV durante o período gestacional.

É importante relatarmos Que Quatro mulheres $(36,3 \%)$ também não possuíam dados na ficha de notificação Que permitissem concluir se elas fizeram uso ou não de ARV durante o pré-natal.

\section{DISCUSSÃO}

A análise dos dados secundários nos possibilitou a identificação de inúmeros entraves na realização de um pré-natal de Qualidade.

Observou-se que o indicador de cobertura de gestantes necessita de melhora em seus índices. Sabe-se que o cadastramento de todas as gestantes no Sisprenatal é efetuado em sua primeira consulta de pré-natal nos centros de saúde, a fim de obterem acesso às consultas de pré-natal subseqüentes. Constatou-se uma grande porcentagem de mulheres Que não realizaram o cadastramento, portanto não realizaram pré-natal nas unidades básicas de saúde.

Essa informação sugere a possibilidade de demanda reprimida para o pré-natal nas UBS, demonstrada em alguns estudos ${ }^{(12-14)}$. Tais achados se opõem ao recomendado pelo Programa de Humanização do Pré-Natal e Nascimento (PHPN), Que possui como um de seus objetivos o cadastramento de todas as gestantes adscritas na área de abrangência de uma unidade básica de saúde, levando a uma cobertura efetiva dessas mulheres, com o inicio da assistência durante o pré-natal, se estendendo ao parto e puerpério $^{(15)}$.

O percentual de cobertura de gestantes não se mostrou adequado, demonstrando Que várias gestantes não realizaram o pré-natal nas UBS, sugerindo a não realização do pré-natal. Conseqüentemente, não ocorreu o repasse de informações à gestante e a solicitação de exames Que possuem o objetivo de prevenir complicações, dentre eles, pode-se citar a realização do teste anti-HIV, com o início de terapia antiretroviral, caso a gestante fosse identificada como soropositiva. Dentro dessa perspectiva, Nishimoto et $\mathrm{al}^{(16)}$ demonstraram Que a realização do pré-natal resulta em redução da transmissão vertical do HIV, pois a gestante obtém informações e acesso a medidas preventivas.
Em relação à captação precoce, esta também não se mostrou adequada. Tanaka et $\mathrm{al}^{(17)}$ realizaram um estudo Que analisou o Programa de Humanização do Pré-Natal e Nascimento em âmbito nacional e concluíram Que a média percentual do indicador de captação precoce na região sudeste é superior a 90\%. No entanto, Quanto maior o porte do município, menor é a média percentual de captação com até 120 dias. Portanto, as capitais brasileiras possuem os menores índices de captação precoce, da ordem de 76,98\%.

Em pesquisa conduzida no ano de 2007 em Porto Velho, Rondônia, 68,6\% das gestantes foram captadas no $1^{\circ}$ trimestre da gestação, demonstrando índices satisfatórios de captação precoce $^{(18)}$. Já em Pernambuco, Recife, outra pesquisa apontou Que apenas $46,6 \%$ das gestantes iniciaram o pré-natal durante o $1^{\circ}$ trimestre de gestação ${ }^{(19)}$. Em nossa pesQuisa, os percentuais obtidos para a capital mineira também seguiram os índices do estudo nacional do PHPN, ou seja, inferiores a 76,98\%.

Tanto em relação à capital mineira Quanto ao Disal, os índices de captação precoce não chegaram a 50\% das gestantes no período analisado. A captação de porcentagem significativa das gestantes em período superior a 16 semanas, o Que configura uma captação 
tardia, impede a detecção precoce de uma possível gestante soropositiva, com a perda do início em tempo adequado de ARV, o Que deveria ocorrer a partir de 14 semanas de gestação.

Em relação ao percentual de gestantes Que realizaram 6 consultas de pré-natal, observamos Que os percentuais de realização se encontram inferiores a $30 \%$. Tanaka et $\mathrm{al}^{\left({ }^{(1)}\right.} \mathrm{em}$ seu inquérito de base populacional sobre o Programa de Humanização do Parto e Nascimento, encontraram no Brasil um percentual de realização de 6 consultas de pré-natal da ordem de 22,6\%, sendo encontrado na região sudeste $21,81 \%$. Como observado, os valores encontrados tanto no Disal Quanto na capital mineira encontram-se superiores aos valores encontrados para a região sudeste no inquérito de base populacional, no entanto, ainda são valores aQuém dos valores esperados.

Tanto no distrito Quanto no município, a maior parte das gestantes não consegue realizar o número mínimo de consultas preconizado pelo PHPN, o Que pode comprometer a Qualidade da assistência prestada durante o pré-natal, reduzindo as possibilidades de prevenção e tratamento da transmissão vertical do HIV.

Segundo o manual de implantação do PHPN, a consulta puerperal deverá ser realizada até 42 dias após a realização do parto $^{(14)}$. Conjugando-se no mesmo indicador o número de 6 consultas de pré-natal e a de puerpério, esses percentuais caem para menos da metade do número de gestantes, tanto no Distrito Leste Quanto em Belo Horizonte.

Sabe-se que o Ministério da Saúde possui recomendações Que visam a prevenção da transmissão vertical do HIV durante o puerpério, através da orientação das mães soropositivas Quanto ao preparo da fórmula infantil para substituição do leite materno, não amamentação e uso contínuo do AZT xarope na criança ${ }^{(20)}$. Como os índices de realização da consulta puerperal estão aQuém do desejado, indicando Que a maioria das mulheres não retorna à UBS até 42 dias após o parto, compromete-se a prevenção contra a transmissão vertical do HIV, na medida em se desconhece se essas mulheres estão amamentando e se a criança está recebendo o AZT xarope.

Em relação ao indicador percentual de gestantes inscritas Que realizaram as 6 consultas de pré-natal e todos os exames básicos, observamos pouca variação nos valores do município. Já o Distrito Leste apresentou crescimento desse indicador, do ano 2004 para 2005 , respectivamente, $17,4 \%$ e $21,9 \%$. Observou-se que menos de $30 \%$ das mulheres conseguem aliar as 6 consultas preconizadas aos exames básicos, comprometendo a Qualidade da assistência pré-natal.

É importante relatar Que o exame anti-HIV não é classificado como exame básico, como não se constitui em exame obrigatório, é analisado em um indicador separado do Que constam os exames básicos. A obrigatoriedade da oferta do teste anti-HIV mediante aconselhamento pré-teste e pós-teste adequados a todas as gestantes é o Que preconiza o ministério da saúde ${ }^{(21)}$.

O percentual de gestantes inscritas Que realizaram o teste antiHIV, também não se mostrou satisfatório; pois tanto no município Quanto no distrito, menos de $50 \%$ das mulheres foram submetidas ao exame anti-HIV durante a gestação nos dois anos consecutivos. Reafirmando nossos achados, Araújo e Nogueira ${ }^{(22)}$ ao realizarem uma pesquisa em uma maternidade pública de referência em Teresina, Piauí, encontraram índices de realização do teste da ordem de $32,4 \%$. Também Nascimento et $\mathrm{al}^{(23)}$ encontraram uma porcentagem peQuena de realização do teste, de apenas 17,6\% na capital Salvador, Bahia. Os dados demonstram Que menos da metade das mulheres foram testadas para o HIV, perdendo oportunidades de identificação de possíveis gestantes soropositivas durante o prénatal.

Como é uma alta porcentagem, sugere-se também a possibilidade de sub-registros. A existência de sub-registros também é evidenciada por Trevisan et $\mathrm{al}^{(24)}$, principalmente na primeira consulta de prénatal, Quando são solicitados os exames básicos. Portanto, o profissional de saúde solicita o exame, no entanto, não ocorre o posterior registro no cartão e na ficha de produção diária das unidades básicas de saúde, o Que pode gerar uma redução no indicador de gestantes Que realizaram o teste anti-HIV. Da mesma forma, também Coutinho et al ${ }^{(25)}$ constataram a ausência de registro da realização do teste anti-HIV em um terço dos cartões de gestante analisados em estudo realizado na cidade de Juiz de Fora, MG.

Nosso estudo demonstrou no perfil das gestantes HIV positivas a persistência de mulheres em idade reprodutiva, na faixa de 25 a 29 anos, e com menos de 8 anos de estudo. Vários estudos têm demonstrado Que justamente essas mulheres com menor grau de instrução e Que dependem dos serviços públicos de saúde são as Que possuem menor probabilidade de serem atendidas, de realizarem o pré-natal com o número de consultas preconizado e a sua captação para o início do pré-natal ocorrer em tempo adequado $^{(26,27)}$.

Em relação ao momento em Que a mulher conheceu seu "status" sorológico, identificou-se uma alta porcentagem de mulheres sabidamente soropositivas para o vírus, antes da gestação $(44,8 \%)$. Corroborando nossos achados, um estudo realizado em Porto Alegre, utilizando as fichas de investigação - Gestante HIV positiva e Crianças Expostas, também constatou ser alta a porcentagem das gestantes Que sabiam de sua soropositividade antes da gestação $(52,7 \%)^{(28)}$. É importante Que sejam estabelecidos fóruns de discussão acerca do direito reprodutivo dessas mulheres, com o objetivo de eualificar os profissionais de saúde para realizarem a abordagem de forma Que se estabeleça o diálogo entre o profissional de saúde e a mulher soropositiva. Essa interação se realizada da forma adequada pode levar a uma consciente tomada de decisões em relação às possibilidades de gestação e à prevenção da transmissão vertical do HIV no pré-natal, parto e puerpério.

Um total de apenas $62,1 \%$ das mulheres soropositivas realizou profilaxia com ARV no período do estudo. A média de idade gestacional de captação para o início do pré-natal não se mostrou satisfatória para a maior parte das gestantes, sendo que apenas as mulheres soropositivas e Que já utilizavam ARV obtiveram início do pré-natal dentro do período preconizado de até 16 semanas. Esse fato demonstra Que as mulheres sabidamente soropositivas provavelmente têm sido orientadas pelos serviços de saúde Quanto à importância do início precoce do pré-natal.

O início dos ARV durante o pré-natal também não se mostrou satisfatório, pois a captação tardia de gestantes levou ao início tardio da terapia antiretroviral, o que aumenta as possibilidades de infecção do concepto. As gestantes identificadas como soropositivas durante o pré-natal foram as Que iniciaram a profilaxia com ARV mais tardiamente, demonstrando as fragilidades do sistema em identificar de forma precoce essas mulheres.

É importante mencionar a Quantidade significativa de gestantes 
soropositivas com informações incompletas em suas fichas de notificação para Que pudéssemos concluir se fizeram uso ou não de ARV durante o pré-natal. O elevado número de campos não preenchidos na ficha de investigação Gestante HIV positiva e crianças expostas, não apenas em relação ao uso de ARV como também em relação à idade, escolaridade, evidência laboratorial do HIV, nos leva a concluir Que há sub-registro de informações.

A existência de sub-registros aliada à contradição nos dados encontrados nas fichas nos leva a concluir que o banco de dados pode não refletir a realidade do atendimento realizado nas UBS. Em estudo realizado por Vasconcelos e Hamann ${ }^{(29)}$, foi demonstrado Que o programa brasileiro de redução de transmissão vertical do HIV se revela frágil, no Que diz respeito à organização, administração e avaliação dos serviços de saúde. Dentre os fatores mencionados estavam a falta de informações adequadas nos prontuários das gestantes, o Que dificultou a avaliação da Qualidade da assistência prestada. As perdas demonstram as fragilidades das informações obtidas com o processamento dos dados, comprometendo a possibilidade de uma caracterização mais detalhada da epidemia, do ponto de vista epidemiológico ${ }^{(30)}$.

\section{CONSIDERAÇÕES FINAIS}

Foram identificados inúmeros entraves à realização de um prénatal de Qualidade na rede básica e Que geram dificuldades na identificação de uma possível gestante soropositiva.
Observamos a existência de protocolos relacionados à execução de condutas adeQuadas na prevenção da transmissão vertical do HIV. Há disponibilidade de antiretrovirais a todas as gestantes, contudo, a identificação tardia de mulheres soropositivas durante o pré-natal resultou no início tardio da terapia, o Que aumenta as chances de infecção do concepto. Observou-se, Que a captação precoce de gestantes é baixa, o Que gera comprometimento da Qualidade da assistência prestada, na medida em Que ocorre a redução do número de consultas durante o pré-natal e de gestantes Que realizaram os exames básicos. A assistência pré-natal não é finalizada no banco de dados Sisprenatal, pois a maioria das gestantes não retorna para a realização da consulta de puerpério.

Deve ser mencionado que os bancos de dados (Sisprenatal e Sinan) podem não retratar de forma autêntica a realidade da atenção pré-natal executada nas unidades básicas. A própria defasagem de tempo, o Que acarreta uma maior atualização do nível local em detrimento do nível central, aliada à presença de sub-registros e à ausência de preenchimento adequado das fichas que alimentam os bancos, compromete a avaliação apenas pelos indicadores do programa. Isso impossibilita a interpretação adequada dos dados.

A partir de um maior aprimoramento das informações fornecidas por esses bancos de dados, o programa direcionado à prevenção da transmissão vertical do HIV terá uma avaliação mais adeQuada. O envolvimento de gestores e a capacitação dos profissionais de saúde Que alimentam esses bancos são medidas essenciais Que fazem parte desse processo.

\section{REFERÊNCIAS}

1. Ministério da Saúde (BR). Casos de Aids notificados no Sinan (número e percentual) em indivíduos menores de 13 anos de idade, segundo categoria de exposição hierareuizada por ano de diagnóstico. Brasil, 1980-2008. Brasília: Ministério da Saúde; 2008.

2. Connor EM, Sperling RS, Gelber R, Kiselev P, Scott G, O'Sullivan $\mathrm{MJ}$, et al. Reduction of maternal infant transmission of human imunodeficiency vírus type I with zidovudine treatment. N Engl I Med 1994; 33 I(18): 1173-80.

3. Kind C, Rudin C, Siegrist CA, Wyler CA, Biedermann K, Lauper $\mathrm{U}$, et al. Prevention of vertical HIV transmission: additive protective effect of elective Cesarean section and zidovudine prophylaxis. Aids 1998; I2(2): 205-10.

4. Mandelbrot L, MD; Chenadec IL, Berrebi A, Bongain A, Bénifla IL; Delfraissy JF, et al. Perinatal HIV-I Transmission: Interaction Between Zidovudine Prophylaxis and Mode of Delivery in the French Perinatal Cohort. JAMA 1998 Jul I; 280(I): 55-60.

5. Ministério da Saúde (BR). Coordenação Nacional de DST/Aids. Recomendações para a profilaxia da transmissão vertical do HIV e terapia antiretroviral em gestantes. Brasília: Ministério da Saúde; 2006.

6. Ministério da Saúde (BR). Secretaria de Vigilância Epidemiológica. Programa Nacional de DST e Aids. A epidemia de HIV-Aids entre crianças e adolescentes. Brasília: Ministério da Saúde; 200I.

7. Ministério da Saúde (BR). Gestante HIV positiva: evento sentinela. Bol Epidemiol Aids 2005; 2(I): 16-2 I.

8. Contandriopoulos AP, Champagne F, Denis IL, Pineault R. Avaliação na área de saúde: conceitos e métodos. In: Hartz
ZMA, organizador. Avaliação em saúde: dos modelos conceituais à prática na análise da implantação de programas. Rio de Janeiro: Fiocruz; 1997. p. 29-47.

9. Ministério da Saúde (BR). Secretaria de Políticas de Saúde. Implantação do Programa de Humanização do Pré-Natal e Nascimento. Brasília: Ministério da Saúde; 2000.

10. Ministério da Saúde (BR). Portaria Técnica Ministerial no 2.325/ GM, de 8 de dezembro de 2003. Define a notificação compulsória da Infecção pelo vírus da imunodeficiência humana (HIV) em gestantes e crianças expostas ao risco de transmissão vertical para todo território nacional. Diário Oficial República 2003 dez.

11. Ministério da Saúde (BR). Portaria Técnica Ministerial no 33/ GM, de 14 de julho de 2005. Inclui doenças à relação de notificação compulsória, define agravos de notificação imediata e a relação dos resultados laboratoriais Que devem ser notificados pelos Laboratórios de Referência Nacional ou Regional. Diário Oficial República 2005 jul.

12. Feliciano KVO, Kovacs MH. Vulnerabilidade programática na prevenção da transmissão materno-fetal da Aids. Rev Bras Saúde Matern Infant 2002; 2(2): 157-65.

13. Leal MC, Gama SGN, Ratto KMN, Cunha CB. Uso do índice de Kotelchuck modificado na avaliação da assistência pré-natal e sua relação com as características maternas e o peso do recémnascido no Município do Rio de Janeiro. Cad Saúde Pública 2004; 20(sup 1): S63-72.

14. Almeida SDM, Barros MBA. Eqüidade e atenção à saúde da gestante em Campinas (SP), Brasil. Rev Panam Salud Publica 2005; 17(1): 15-25. 
15. Ministério da Saúde (BR). Secretaria de Políticas de Saúde. Implantação do Programa de Humanização do Pré-Natal e Nascimento. Brasília: Ministério da Saúde; 2000.

16. Nishimoto TMI, Eluf NJ, Rozman MA. Transmissão maternoinfantil do vírus da imunodeficiência humana: avaliação de medidas de controle no município de Santos. Rev Assoc Med Bras 2005; 5 I (I): 54-60.

17. Tanaka OY,Tanaka ACA, Lima FD, Marucci D, Carvalho CAC, Locatelli C, et al. Projeto de avaliação nacional do Programa de Humanização do Pré-Natal e Nascimento (PHPN). São Paulo: Ministério da Saúde/UNESCO; 2004.

18. Santos MAM, Moreira KFA. Avaliação da Qualidade da Assistência Pré-Natal em Porto Velho-RO. In: III Mostra de Produções em Saúde da Família; 2006; Brasília(DF), Brasil. Brasília: Ministério da Saúde; 2006.

19. Carvalho VCP. Fatores associados à realização de pré-natal não adequado: estudo das puérperas atendidas em dois hospitais de referência para gravidez de alto risco do SUS em Recife [dissertação]. Recife: Departamento de Medicina Social, Universidade Federal de Pernambuco; 2005.

20. Barroso LMM, Galvão MTG. Avaliação de atendimento prestado por profissionais de saúde a puérperas com HIV/Aids. Texto Contexto Enferm 2007; 16(3): 463-9.

21. Ministério da Saúde (BR). Coordenação Nacional de DST e Aids. Secretaria de Políticas de Saúde. Aconselhamento em DST, HIV e Aids: diretrizes e procedimentos básicos. Brasília: Ministério da Saúde; 2000.

22. Araújo LM, Nogueira LT. Transmissão vertical do HIV: situação encontrada em uma maternidade de Teresina. Rev Bras Enferm 2007; 60(4): 396-9.
23. Nascimento ER, Paiva MS, Rodrigues QP. Avaliação da cobertura e indicadores do Programa de Humanização do Pré-natal e Nascimento no município de Salvador, Bahia, Brasil. Rev Bras Saude Mater Infant 2007; 7(2): 191-7.

24. Trevisan MR, De Lorenzi, DRS, Araújo NM, Ésber K. Perfil da assistência pré-natal entre usuárias do Sistema Único de Saúde em Caxias do Sul. Rev Bras Ginecol Obstet 2002; 24(5): 293-9.

25. Coutinho T, Teixeira MTB, Dain S, Sayd ID, Coutinho LM. AdeQuação do processo de assistência pré-natal entre as usuárias do Sistema Único de Saúde em Juiz de Fora - MG. Rev Bras Ginecol Obstet 2003; 25(10): 717-24.

26. Travassos C, Viacava F, Fernandes C, Almeida CM. Desigualdades geográficas e sociais na utilização de serviços de saúde no Brasil. Ciênc Saúde Coletiva 2000; 5(1): 133-49.

27. Coimbra LC, Silva AAM, Mochel EG, Alves MTSSB, Ribeiro VS, Aragão VMF, et al. Fatores associados à inadeQuação do uso da assistência pré-natal. Rev Saúde Pública 2003; 37(4): 456-62.

28. Torres SR, Luz AMH. Gestante HIV positiva e crianças expostas: estudo epidemiológico da notificação compulsória. Rev Gaúcha Enferm 2007; 28(4): 505- I I.

29. Ministério da Saúde (BR). Vasconcelos ALR, Hamann EM. Por Que o Brasil ainda registra elevados coeficientes de transmissão vertical do HIV? Secretaria de Vigilância em Saúde. Programa Nacional de DST e Aids. Rev Bras Saúde Matern Infant 2005; 5(4): 483-92.

30. Cruz MM, Toledo LM, Santos EM. O sistema de informação de Aids do Município do Rio de Janeiro: suas limitações e potencialidades enquanto instrumento da vigilância epidemiológica. Cad Saúde Pública 2003; 19(1): 81-9. 